\title{
Characteristics of spinal dissemination in adult low-grade glioma: a retrospective cohort study at a single institute
}

\author{
Jianxin Chen", Qi Shi", Shan Li, Yuze Zhao, Hongyan Huang^ \\ Department of Oncology, Beijing Shijitan Hospital of Capital Medical University, Beijing, China \\ Contributions: (I) Conception and design: J Chen, H Huang; (II) Administrative support: H Huang; (III) Provision of study materials or patients: \\ J Chen, Q Shi; (IV) Collection and assembly of data: J Chen, Q Shi, S Li, Y Zhao; (V) Data analysis and interpretation: Q Shi, H Huang; (VI) \\ Manuscript writing: All authors; (VII) Final approval of manuscript: All authors. \\ \#These authors contributed equally to this work. \\ Correspondence to: Hongyan Huang. Department of Oncology, Beijing Shijitan Hospital of Capital Medical University, Beijing, China. \\ Email: huangh1975@mail.ccmu.edu.cn.
}

Background: In adults, metastatic spinal dissemination (MSD) in low-grade glioma (LGG) is an unusual
phenomenon of the central nervous system. This study sought to investigate the clinical characteristics of
adult MSD in LGG and its course.
Methods: A retrospective analysis was performed from June 2011 to July 2021 in adult LGG patients with
MSD treated at the Oncology Department of the Beijing Shijitan Hospital, Capital Medical University. The
time to MSD and overall survival (OS) were estimated using Kaplan-Meier plotting. A univariate analysis was
performed using a logarithmic-rank test, and a multivariate analysis was performed using Cox proportional
hazards models.

Results: Thirteen adult LGG patients with MSD were enrolled in this retrospective study. Most of the primary tumors were grade II $(92.3 \%)$ or astrocyte derived $(7.7 \%)$. The median total OS time from the initial surgery, the time to MSD and the OS time after MSD of the 13 adult LGG patients was 45.0, 15, and 16 months, respectively.

Conclusions: Adult LGG patients with MSD had a poor prognosis. Enhanced magnetic resonance imaging was highly recommended in adult LGG patients. Maybe parts of patients appeared to experience prolonged clinical benefits from systemic salvage chemotherapy and intrathecal injection after MSD. Novel modalities need to be explored to improve the outcomes of patients with MSD.

Keywords! Low-grade glioma (LGG); metastatic spinal dissemination (MSD); survival; clinical characteristics

Submitted Oct 29, 2021. Accepted for publication Dec 16, 2021.

doi: $10.21037 / \mathrm{apm}-21-3390$

View this article at: https://dx.doi.org/10.21037/apm-21-3390

\section{Introduction}

Low-grade gliomas (LGGs) occur primarily in children and young to intermediate age adults and are classified as grade I and II tumors under the World Health Organization (WHO) classification system (1-3). LGGs in adults have highly variable clinical behaviors that are not easy to predict based on histological class $(4,5)$. Most adult LGGs are indolent, and patients usually have an excellent probability of long-term survival under the current treatment approaches, but some adult LGGs progress rapidly to glioblastoma (GBM) (6,7). According to currently available data, the distinction between indolent and rapid progress has not yet been achieved. We further clarify the general clinical characteristics of adult low-grade gliomas in China.

^ ORCID: 0000-0001-8340-6660. 
According to currently research and our clinical observations, the most common metastasis of glioma is intracranial metastasis or recurrence in situ. Generally, the metastatic spinal dissemination (MSD) of malignant glioma is rare, and only a few studies have indicated that metastatic spread appears to be more common in GBM than in LGG (8). In children, spinal dissemination often occurs in pilocytic astrocytoma (WHO, grade I), and is more common in GBM in adults $(9,10)$. With the development of comprehensive diagnostic methods and treatments, including magnetic resonance imaging (MRI) and chemotherapy, the prognosis for GBM has improved. Thus, the number of cases reported on spinal glioma dissemination has gradually increased. However, for spinal dissemination in adult LGG, limited data are available on its true incidence, presentation, characteristics, outcomes, and best management techniques. In clinical practice, these gaps have led to uncertain conclusions about the outcomes of adult patients with LGG MSD. In addition, the findings of most previous studies were derived from a small data set $(<10$ cases $)$ and case reports.

Thus, we performed a retrospective analysis of LGG patients with MSD over 10 years at our clinic. Based on the historical data, the results showed that adult LGG patients with MSD had a much worse prognosis than those without dissemination. Effective treatments need to be explored to improve survival. We present the following article in accordance with the STROBE reporting checklist (available at https://dx.doi.org/10.21037/apm-21-3390).

\section{Methods}

\section{Patients and study design}

According to the World Health Organization's classification (11) of primary intracranial tumors, LGGs contain with grade I tumors, which contain none of the histologic features, and grade II tumors, characterized by the presence of cytologic atypia alone. Low-grade astrocytic tumors include diffuse astrocytoma, pilomyxoid astrocytoma, and pleomorphic xanthoastrocytoma (WHO grade II), as well as subependymal giant cell astrocytoma (SEGA) and pilocytic astrocytoma (WHO grade I tumors). Thirteen adults (aged $\geq 16$ years) with MSD in LGG were treated at Beijing Shijitan Hospital from June 2011 to July 2021. Demographic, treatment, and survival data were retrospectively collected. Pediatric LGGs were excluded due to their distinct molecular pathogenesis (12), and patients with extracranial dissemination other than spinal dissemination were also excluded. The pathological diagnosis of LGG was histologically confirmed, and spinal dissemination was radiologically diagnosed using enhancedspine MRI with or without positive cerebrospinal fluid (CFS) for tumor cells by at least 2 radiologists. Patients were followed up with routinely by telephone or outpatient visits. The WHO 2007 classification and response assessment in neuro-oncology criteria (13) were adopted for the pathological diagnosis and response assessment criteria. We provided representative images to identify MSD about cervical, thoracic, and lumber spinal spread in Figure 1.

\section{Statistical analysis}

Overall survival (OS) and total OS were defined as the interval from MSD and initial surgery to death from any cause or the last follow-up visit, respectively. Time to MSD (TTMSD) was defined as the time from the initial surgery to the diagnosis of spinal dissemination based on the results of an enhanced MRI or CSF examination. OS and TTMSD were estimated using Kaplan-Meier plotting. The univariate analysis was conducted using the logarithmic-rank test. The multivariate analysis was conducted using the Cox proportional hazards model. All analyses were performed with SPSS (version 23, IBM Corporation, Armonk, NY, USA). A P value $<0.05$ was considered statistically significant.

\section{Ethical statement}

All procedures performed in this study involving human participants were in accordance with the Declaration of Helsinki (as revised in 2013). The study was approved by institutional ethics board of Beijing Shijitan Hospital [No. sjtky11-lx-2021(64)]. Individual consent for this retrospective analysis was waived.

\section{Results}

\section{Patient characteristics}

From June 2011 to July 2021, 13 adult patients with LGG MSD were enrolled in this retrospective study. The characteristics of these 13 patients are summarized in Table 1. Of the 13 patients, 8 were male, and 5 were female. The patients had a median age of 33.0 years (range: 17-66 years). Most of the primary tumors were grade II 

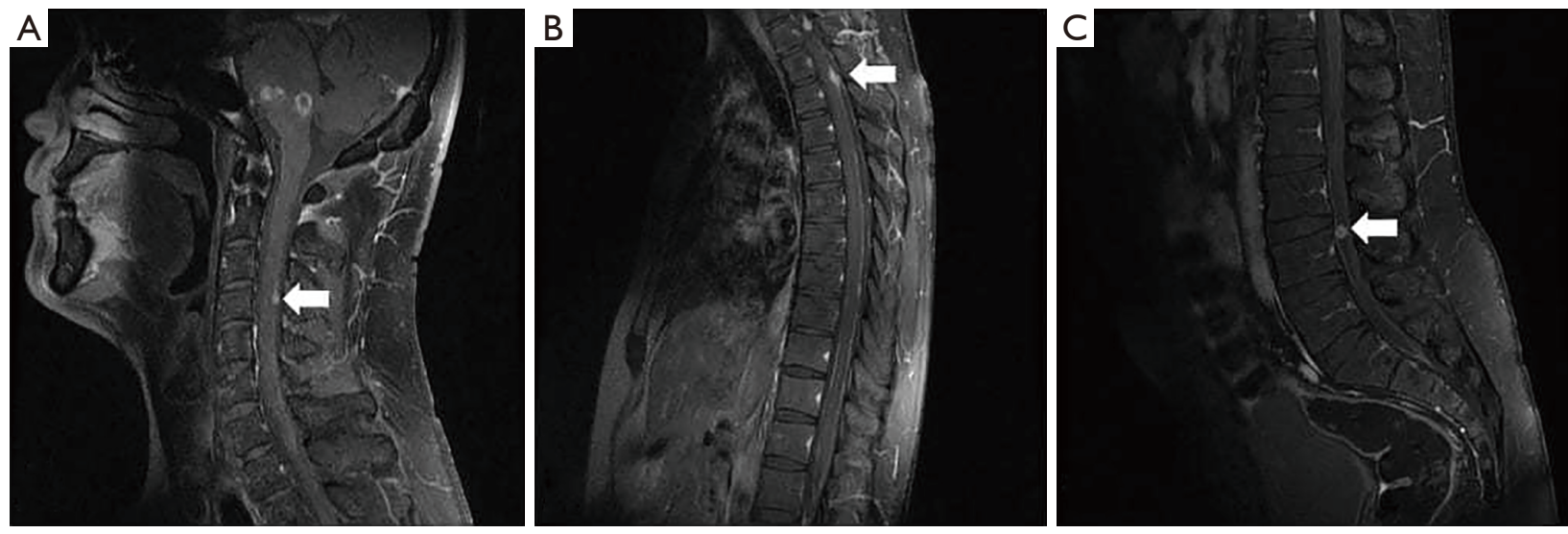

Figure 1 Three patients in this study with metastatic spinal dissemination about cervical, thoracic, and lumber spinal spread, respectively. The white arrows indicate the magnetic resonance imaging findings of metastatic spinal dissemination. (A) Male, 32 years old, primary lowgrade glioma in brainstem, metastatic spinal dissemination with cervical spinal spread. (B) Male, 44 years old, primary low-grade glioma in right temporal, metastatic spinal dissemination with thoracic spinal spread. (C) Male, 33 years old, primary low-grade glioma in left temporal, metastatic spinal dissemination with lumber spinal spread.

Table 1 Patient demographic characteristics

\begin{tabular}{|c|c|c|c|c|c|c|c|}
\hline Pt. No. & Sex & Age (years) & Pathology & Primary tumor location & Surgery & Adjuvant radiotherapy [Gy] & Adjuvant chemotherapy [cycles] \\
\hline 1 & M & 19 & PA & B (Sellar region) & PR & CSI [36] & 0 \\
\hline 2 & M & 17 & OA & B (Multiple) & Biopsy & - & Others \\
\hline 3 & M & 33 & DA & S (Sacrococcygeal) & Biopsy & - & $\mathrm{TMZ}[5]$ \\
\hline 4 & M & 44 & DA & B (R Tempora) & GTR & CCRT [60] & $\mathrm{TMZ}[3]$ \\
\hline 6 & $\mathrm{~F}$ & 41 & DA & B (L Frontotemporal) & Biopsy & CCRT [45] & TMZ [12] \\
\hline 7 & M & 33 & DA & B (L Tempora) & PR & $\mathrm{RO}[60]$ & - \\
\hline 8 & $\mathrm{~F}$ & 33 & DA & B (L Frontal) & GTR & CCRT [60] & $\mathrm{TMZ}$ [13] \\
\hline 9 & $\mathrm{~F}$ & 41 & DA & B (L Tempora) & GTR & CCRT [60] & TMZ [6] \\
\hline 13 & $\mathrm{~F}$ & 66 & OA & B (L Temporal) & GTR & CCRT [60] & TMZ [10] \\
\hline
\end{tabular}

Pt, patient; No., number; M, male; F, female; PA, pilocytic astrocytoma; DA, diffuse astrocytoma; OA, oligoastrocytoma; B, brain; S, spine; L, left; R, right; PR, partial resection; GTR, gross-total resection; RO, radiotherapy only; TMZ, temozolomide; NCNU, amino-ethylenenitrourea, 5-fluorouracil, prednisolone; CSI, craniospinal irradiation; CCRT, concurrent chemoradiation.

$(92.3 \%)$ and astrocyte derived $(7.7 \%)$. Further, $76.9 \%$ of the patients had intracranially located primary tumors. All patients underwent surgery or biopsy to obtain the histology of the primary tumor. $76.9 \%$ of patients received adjuvant radiology therapy, and $61.5 \%$ received adjuvant chemotherapy after surgery.

\section{Clinical manifestations of MSD}

The most common locations of metastasis dissemination 
Table 2 Site and type of metastatic disease

\begin{tabular}{lcccc}
\hline Pt. No. & Time to MSD (months) & Symptoms at spinal dissemination & Dissemination location & Systemic salvage chemotherapy [cycles] \\
\hline 1 & 0.2 & MRI & C & MTX \\
2 & 0.9 & Epilepsy & TSC & DDP [1] \\
3 & 0.9 & Limb weakness & L/S & TMZ [5] \\
4 & 6.0 & Pain & L/S & Bevacizumab + CCNU [3], CE [1] \\
5 & 35.0 & Headache & TSC & CE [4] \\
6 & 15.0 & Pain & C & C [5], CE + CTX [13], CE + TMZ [2] \\
7 & 14.0 & Pain & TSC & - \\
8 & 25.0 & Limb weakness & TSC & DDP+VM-26 [4], ACNU, TMZ [3] \\
9 & 64.0 & MRI & TSC & CSI DDP + VM-26 [5] \\
10 & 24.0 & Limb weakness & C & DDP + VM-26 [5], CGA [4] \\
11 & 3.0 & MRI & TSC & DDP [1] \\
12 & 40.0 & 36.0 & Limb weakness & TSC \\
13 & Unawareness & TSC & TMZ [16], TMZ [9]
\end{tabular}

MRI, magnetic resonance imaging; TSC, total spinal cord; L/S, lumbar and sacral cord; C, cervical cord; MTX, methotrexate; DDP, cisplatin; CTX, cerebrotendinous xanthomatosis; CE, carboplatin etoposide; CCNU, Lomustine; VM-26, teniposide; ACNU, Nimustine; CSI, craniospinal irradiation; TMZ, Temozolomide; CGA, chlorogenic acid.

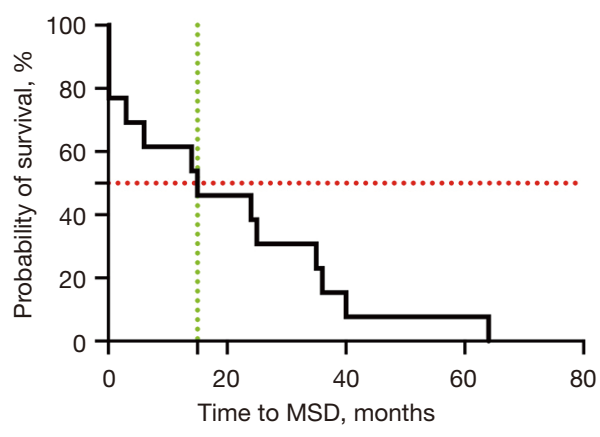

Figure 2 Median time to metastatic spinal dissemination (15 months). A Kaplan-Meier analysis was used to calculate the median time.

were the entire spine (61.5\%), the cervix $(23.1 \%)$, and other sites (15.4\%). After spinal dissemination, 84.6\% (11/13) of the patients presented with neurological symptoms suggestive of myelopathy, including weakness of the limbs [3], pain of the limbs, lumbar, and back [3], headache and dizziness [2], epilepsy [1], numbness of the limb [1], and a lack of awareness [1]. Two of the patients did not show any obvious symptoms, and spinal dissemination was found routinely on MRI (Table 2).

The estimated median TTMSD in our cohort was
15 months (0-64.0 months) (see Figure 2). In 1 patient, MSD was only found 6 days after the initial surgery with a primary tumor in the sellar region and concurrent spinal dissemination in the suprasellar region.

No significant associations were detected in the univariate Cox proportional hazard models between age, sex, pathology, primary tumor location, or TTMSD.

\section{Overall survival}

Following spinal dissemination, $12(92.3 \%)$ patients received systemic salvage chemotherapy, and 3 (23.1\%) patients received radiotherapy ( 2 received craniospinal irradiation, and 1 received local radiotherapy). All patients who underwent systemic chemotherapy were treated with intrathecal methotrexate.

As of July 2021, 2 (15.4\%) patients were still alive and had a median follow-up time of 54 months (22-86 months), and 11 patients (84.6\%) had died due to disease progression. The median total OS of this cohort was 45 months (10-86 months), which is shorter than that of LGG without spinal dissemination according to historical data (14), and the median OS was 16 months (0.5-85 months) (see Figure 3).

No significant associations were detected in the 


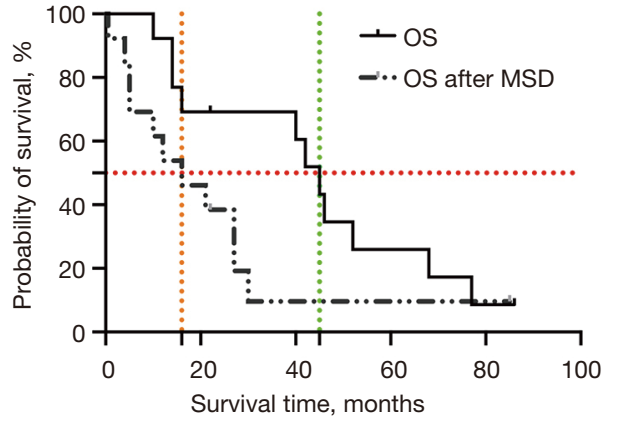

Figure 3 Overall survival (45 months) and median survival after metastatic spinal dissemination (16 months) of 13 adult low-grade glioma patients with metastatic spinal dissemination. A KaplanMeier analysis was used to calculate overall and median survival.

univariate Cox proportional hazard models between age, sex, pathology, primary tumor location, TTMSD, treatment, OS, or total OS.

\section{Discussion}

The spread and transfer of malignant glioma MSD along the nerve axis is rare. MSD can occur in patients with high-grade glioma and LGG, and many patients will simultaneously suffer from spinal dissemination and intracranial lesions (15). Stanley et al. concluded that the probability of spinal metastasis in 600 cases of GBM was $2 \%$ (16), and Chamdine et al. reported that the probability of spinal metastasis at the time of diagnosis and the progression of LGG was $2-5 \%$ and $5-12 \%$, respectively (17).

In our retrospective study, of 282 adult LGG patients treated at our clinic over a 9-year period, 13 had spinal dissemination. The incidence rate of spinal dissemination after LGG resection was 5.6\%, which was lower than the reality, considering that the follow-up of some patients was lost.

Despite the small sample size, the location and dissemination symptoms were well documented in this study. In relation to the location of tumor spread, spreading throughout the spinal cord was predominant and was observed in 10 patients, while spreading in the cervical spine was only observed in 3 patients. These results are consistent with those of previous reports and indicate that spinal dissemination can occur in all regions of the spine. No predilection for any region was noted, and the involvement of the entire spine was not uncommon.

LGG has a slow progression, a long survival period (18), and a low probability of MSD. The symptoms of spinal cord injury appear late. Generally, a diagnosis of MSD is only considered when symptoms of spinal cord and/ or nerve root injury are observed. Due to a lack of clear symptoms of spinal cord injury or clinical negligence, some patients are misdiagnosed or not diagnosed. In this study, while most of the patients had related symptoms at dissemination, 4 patients who were diagnosed with MSD were asymptomatic, which suggests that this complication may occur occultly to some extent. Enhanced MRI showed high sensitivity in detecting spine dissemination, but the question of whether MRI should be used to detect spine dissemination without symptoms requires further prospective study.

LGGs are indolent and have a long survival period (18); however, patient prognosis becomes poor once there is a diagnosis of spinal dissemination. In this cohort, the median OS of adults with MSD in LGG was only 52 months. Conversely, in the RTOG 9802 study, the median OS of LGG patients with unfavorable factors who received radiation therapy after surgery was at least 7.6 years. In this study, the interval was short in terms of survival time after spinal dissemination, with a median time of only 20.2 months.

A novel modality should be explored to improve the prognosis of spinal dissemination (19). In this study almost all the patients received salvage chemotherapy; however, 4 patients received radiation therapy for MSD. Notably, no significant differences were detected between the outcomes of the radiation therapy patients and another patient after MSD.

The limited sample size may be one of the main reasons for the lack of standard recommendations for spinal dissemination. Notably, a female patient with a primary astrocytoma tumor (grade 2) survived $>30$ months after MSD and was diagnosed 15 months after initial resection. In our clinic, this patient received combined chemotherapy with carboplatin and etoposide for 12 cycles after spinal dissection and fully consented to this nonstandard treatment. Further, the neurological symptoms related to myelopathy were relieved after chemotherapy. This case suggests that systemic chemotherapy contributes to prolonged survival; however, this finding should be validated in a well-designed study with a larger sample size in the future.

Several factors limited this retrospective research, including its small sample size and a lack of uniformity in the treatments administered after spinal dissemination. A small number of the sample reached the survival event, and no predictive factor was identified as affecting the total OS or OS after MSD. Non-uniform treatments after spinal 
dissection may inevitably lead to different outcomes in MSD patients. Consequently, our conclusions should be interpreted with caution.

Our study showed that adult LGG patients with MSD had a much poor prognosis; however, there was no clear evidence that salvage therapy prolongs the survival time of MSD patients. We suggested enhanced MRI for the diagnosis. Despite this, a small proportion of patients appeared to experience prolonged clinical benefits from systemic salvage chemotherapy and intrathecal injection after spinal dissemination, and novel modalities should be explored to improve the outcomes of patients with MSD.

\section{Acknowledgments}

Funding: This work was funded by the National Natural Science Foundation of China (81572799).

\section{Footnote}

Reporting Checklist: The authors have completed the STROBE reporting checklist. Available at https://dx.doi. org/10.21037/apm-21-3390

Data Sharing Statement: Available at https://dx.doi. org/10.21037/apm-21-3390

Conflicts of Interest: All authors have completed the ICMJE uniform disclosure form (available at https://dx.doi. org/10.21037/apm-21-3390). The authors have no conflicts of interest to declare.

Ethical Statement: The authors are accountable for all aspects of the work in ensuring that questions related to the accuracy or integrity of any part of the work are appropriately investigated and resolved. All procedures performed in this study involving human participants were in accordance with the Declaration of Helsinki (as revised in 2013). The study was approved by institutional ethics board of Beijing Shijitan Hospital [No. sjtky11-lx-2021(64)]. Individual consent for this retrospective analysis was waived.

Open Access Statement: This is an Open Access article distributed in accordance with the Creative Commons Attribution-NonCommercial-NoDerivs 4.0 International License (CC BY-NC-ND 4.0), which permits the noncommercial replication and distribution of the article with the strict proviso that no changes or edits are made and the original work is properly cited (including links to both the formal publication through the relevant DOI and the license). See: https://creativecommons.org/licenses/by-nc-nd/4.0/.

\section{References}

1. Sanai N, Chang S, Berger MS. Low-grade gliomas in adults. J Neurosurg 2011;115:948-65.

2. Diwanji TP, Engelman A, Snider JW, et al. Epidemiology, diagnosis, and optimal management of glioma in adolescents and young adults. Adolesc Health Med Ther 2017;8:99-113.

3. Funakoshi Y, Hata N, Kuga D, et al. Pediatric Glioma: An Update of Diagnosis, Biology, and Treatment. Cancers (Basel) 2021;13:758.

4. Cancer Genome Atlas Research Network; Brat DJ, Verhaak RG, et al. Comprehensive, Integrative Genomic Analysis of Diffuse Lower-Grade Gliomas. N Engl J Med 2015;372:2481-98.

5. Yang $\mathrm{Q}$, Xiong $\mathrm{Y}$, Jiang $\mathrm{N}$, et al. Integrating Genomic Data with Transcriptomic Data for Improved Survival Prediction for Adult Diffuse Glioma. J Cancer 2020;11:3794-802.

6. Hanif F, Muzaffar K, Perveen K, et al. Glioblastoma Multiforme: A Review of its Epidemiology and Pathogenesis through Clinical Presentation and Treatment Asian Pac J Cancer Prev 2017;18:3-9.

7. Zhang $\mathrm{M}, \mathrm{Xu} F, \mathrm{Ni} W$, et al. Survival impact of delaying postoperative chemoradiothera-py in newly-diagnosed glioblastoma patients. Transl Cancer Res 2020;9:5450-8.

8. Mariniello G, Peca C, Del Basso De Caro M, et al. Brain gliomas presenting with symptoms of spinal cord metastasis. Neuroradiol J 2015;28:478-82.

9. Sievert AJ, Fisher MJ. Pediatric low-grade gliomas. J Child Neurol 2009;24:1397-408.

10. Bandopadhayay P, Bergthold G, London WB, et al. Long-term outcome of 4,040 children diagnosed with pediatric low-grade gliomas: an analysis of the Surveillance Epidemiology and End Results (SEER) database. Pediatr Blood Cancer 2014;61:1173-9.

11. Louis DN, Perry A, Wesseling P, et al. The 2021 WHO Classification of Tumors of the Central Nervous System: a summary. Neuro Oncol 2021;23:1231-51.

12. Ryall S, Tabori U, Hawkins C. A comprehensive review of paediatric low-grade diffuse glioma: pathology, molecular genetics and treatment. Brain Tumor Pathol 2017;34:51-61.

13. Chukwueke UN, Wen PY. Use of the Response 
Assessment in Neuro-Oncology (RANO) criteria in clinical trials and clinical practice. CNS Oncol 2019;8:CNS28.

14. Shaw EG, Wang M, Coons SW, et al. Randomized trial of radiation therapy plus procarbazine, lomustine, and vincristine chemotherapy for supratentorial adult lowgrade glioma: initial results of RTOG 9802. J Clin Oncol 2012;30:3065-70.

15. Verma N, Nolan C, Hirano M, et al. Intramedullary spinal cord and leptomeningeal metastases from intracranial lowgrade oligodendroglioma. Clin Imaging 2014;38:505-7.

16. Stanley P, Senac MO Jr, Segall HD. Intraspinal seeding from intracranial tumors in children. AJR Am J

Cite this article as: Chen J, Shi Q, Li S, Zhao Y, Huang H. Characteristics of spinal dissemination in adult low-grade glioma: a retrospective cohort study at a single institute. Ann Palliat Med 2021;10(12):12643-12649. doi: 10.21037/apm-213390
Roentgenol 1985;144:157-61.

17. Chamdine O, Broniscer A, Wu S, et al. Metastatic LowGrade Gliomas in Children: 20 Years' Experience at St. Jude Children's Research Hospital. Pediatr Blood Cancer 2016;63:62-70.

18. Claus EB, Walsh KM, Wiencke JK, et al. Survival and low-grade glioma: the emergence of genetic information. Neurosurg Focus 2015;38:E6.

19. Conti A, Acker G, Kluge A, et al. Decision Making in Patients With Metastatic Spine. The Role of Minimally Invasive Treatment Modalities. Front Oncol 2019;9:915. 